\title{
Diagnóstico de enfermagem risco de úlcera por pressão segundo taxonomia II da North American Nursing Diagnosis Association
}

\section{Nursing diagnosis ulcer risk of pressure by the North American Nursing Diagnosis Association taxonomy II}

\section{Diagnóstico de enfermería del riesgo de la úlcera por presión de acuerdo a la North-American Nursing Diagnosis Association}

\author{
Belarmino Santos de Sousa Júnior', Ana Elza Oliveira de Mendonça², Fernando Hiago da Silva Duarte², \\ Cíntia de Carvalho Silva ${ }^{3}$
}

\begin{abstract}
RESUMO
Objetivo: Identificar a prevalência dos fatores de risco para o diagnóstico de enfermagem (DE) de risco de úlcera por pressão (UP) de acordo com a taxonomia II da North American Nursing Diagnosis Association (NANDA-I) em pacientes na unidade de terapia intensiva (UTI). Métodos: Estudo transversal de natureza quantitativa realizado em um hospital da rede privada de saúde, no período de janeiro a maio de 2014. O projeto foi avaliado e aprovado pelo Comitê de Ética em Pesquisa. Participaram do estudo 30 pacientes. Os dados foram coletados diariamente durante o exame físico por meio de um instrumento semiestruturado. Resultados: Elencaram-se 20 fatores de riscos. Os mais frequentes foram: escore na Escala de Braden (EB) <18, agentes farmacológicos, alteração na função cognitiva, atrito em superfície, déficit do autocuidado, forças de cisalhamento, incontinência, nutrição inadequada, pressão sobre proeminência óssea, redução na mobilidade, redução na perfusão tissular e umidade da pele. Conclusão: A identificação dos fatores de risco de UP reforça a importância da implementação do processo de enfermagem (PE) a esses pacientes, colaborando com o planejamento das ações do enfermeiro fundamentadas e adequadas de acordo com as necessidades de cada indivíduo.
\end{abstract}

DESCRITORES: Úlcera por pressão. Diagnóstico de enfermagem. Cuidados de enfermagem. Estomaterapia.

\begin{abstract}
Objective: To identify the prevalence of risk factors for the nursing diagnosis of pressure ulcer (PU) risk in accordance with the taxonomy II of the North American Nursing Diagnosis Association (NANDA-I) in patients in the intensive care unit (ICU). Methods: Cross-sectional quantitative study conducted in a hospital of the private health system, from January to May 2014. The project was approved by the Research Ethics Committee. The study included 30 patients; data were collected daily during physical examination by a semi-structured instrument. Results: Twenty risk factors in total were listed, with the most frequent being: score on the Braden Scale (BS) <18, pharmacological agents, change in cognitive function, surface friction, self-care deficit, shear forces, incontinence, inadequate nutrition, pressure on bony prominence, reduced mobility, reduced tissue perfusion and skin moisture. Conclusion: The identification of pressure ulcer risk factors reinforces the importance of the implementation of the nursing process in these patients, collaborating with the planning of reasoned and adequate nurse's actions according to the needs of each individual.
\end{abstract}

DESCRIPTORS: Pressure ulcer. Nursing diagnosis. Nursing care. Stomatherapy.

\footnotetext{
'Universidade Potiguar (UnP) - Natal (RN), Brasil. Endereço para correspondência: Rua Barão de Lucena, 62, Torre 2, Apto. 1703 - CEP: 59066 -285 Natal (RN), Brasil - E-mail: sousajunyor@gmail.com

¿Universidade Federal do Rio Grande do Norte (UFRN) - Natal (RN), Brasil.

universidade de Pernambuco (UPE) - Caruaru (PE), Brasil.

Artigo recebido em: 29/02/2016 - Aceito para publicação em: 29/03/2016
} 


\section{RESUMEN}

Objetivo: Identificar la prevalencia de los factores de riesgo para el diagnóstico de enfermería del riesgo de úlcera por presión, de acuerdo a la taxonomía II de la North-American Nursing Diagnosis Association (NANDA-I) en pacientes en la unidad de cuidados intensivos. Métodos: Estudio cuantitativo trasversal realizado en un hospital del sistema privado de salud, en el período desde enero hasta mayo de 2014. El proyecto fue evaluado y aprobado por el Comité de Ética en Investigación. El estudio incluyó a 30 pacientes, y se recogieron datos todos los días durante el examen físico por medio de un instrumento semiestructurado. Resultados: Se enumeran hasta 20 factores de riesgos, y los más frecuentes fueron: puntuación en la Escala de Braden <18, agentes farmacológicos, cambio en la función cognitiva, fricción superficial, déficit del autocuidado, fuerzas de corte, incontinencia, nutrición inadecuada, presión sobre la prominencia ósea, movilidad reducida, perfusión tisular reducida y humedad de la piel. Conclusión: La identificación de los factores de riesgo de la úlcera por presión refuerza la importancia de la aplicación del proceso de enfermería en estos pacientes, lo que colabora con la planificación de las acciones de enfermería motivadas y adecuadas de acuerdo a las necesidades de cada individuo.

DESCRIPTORES: Úlceras por presión. Diagnóstico de enfermería. Cuidados de enfermería. Estomaterapia.

\section{INTRODUÇÃO}

A discussão sobre a qualidade da assistência à saúde é algo presente em âmbito nacional e internacional, desde a década de 1980, em razão do envelhecimento global da população, dos escassos recursos disponíveis e dos altos custos para manutenção dos serviços. Assim, para os gerentes de enfermagem e administradores de serviços de saúde, as úlceras por pressão (UPs) representam um grave problema e um desafio à equipe, em termos de sofrimento pessoal e econômico, uma vez que consomem demasiadas horas de enfermagem e recursos do sistema de saúde ${ }^{1}$.

O desenvolvimento das UPs está etiologicamente associado a dois fatores determinantes, a duração e a intensidade da pressão. Tais lesões ocorrem quando a área afetada sofre morte celular, caracterizada pela compressão entre uma superfície rígida e uma proeminência óssea por um período de tempo prolongado. Outros fatores potencializam a sua ocorrência, como os fatores intrínsecos e extrínse$\cos ^{2}$. Aos fatores intrínsecos ao paciente elencam-se a idade, a imobilidade, o estado nutricional, as anemias, as infecções, as incontinências e a sensibilidade cutânea. Já no que concerne aos fatores extrínsecos, decorrentes do ambiente, destacam-se: cisalhamento do lençol, umidade, higiene deficiente, ausência de mudança de decúbito, uso de instrumentos ortopédicos, sondas fixadas de forma inadequada, uso incorreto de agentes físicos e químicos, tipos de colchões e assentos inadequados ${ }^{3}$.

Assim, compreende-se que as UPs são uma das complicações frequentes em pacientes críticos internados em unidade de terapia intensiva (UTI), uma vez que estes estão expostos a fatores de risco para seu desenvolvimento, como: idade, déficit no estado nutricional, estado geral comprometido, inconstância hemodinâmica, limitação da mobilidade, decorrentes de patologias diversas ou sequelas destas ${ }^{4}$.

No ambiente de terapia intensiva, a enfermagem possui uma metodologia própria de trabalho, fundamentada em um processo sistemático para proporcionar o cuidado ao paciente. $\mathrm{O}$ processo de enfermagem (PE) é definido por uma conduta deliberada de resolução de problema para atender às necessidades de enfermagem e de cuidados de saúde das pessoas. Esse processo é formado pelas seguintes etapas: histórico; diagnósticos de enfermagem (DEs); planejamento das ações; implementação; evolução ${ }^{5}$.

No PE, a etapa DEs envolve a avaliação clínica das respostas do paciente ou dos grupos aos problemas de saúde e fornece a base para as intervenções do enfermeiro, objetivando atingir os resultados esperados. Por meio do raciocínio clínico referente os dados coletados por intermédio de um roteiro de entrevista e de exame físico, é possível identificar os diagnósticos de enfermagem ${ }^{5}$. No que diz respeito à classificação dos diagnósticos de enfermagem, a North American Nursing Diagnosis Association (NANDA-I) tem contribuído para o crescimento e aperfeiçoamento desses diagnósticos, como também para o desenvolvimento de um sistema com vistas a classificá-los em uma taxonomia ${ }^{6}$.

Assim, destaca-se o PE como o método científico inerente à profissão, direcionado pela Sistematização da Assistência de Enfermagem (SAE), que organiza e respalda o trabalho do enfermeiro ${ }^{7}$. Além disso, a SAE visa atender à Resolução 358/2009, do Conselho Federal de Enfermagem (COFEN), porém sua 
implementação ainda é um ideal a ser alcançado, mesmo sabendo dos requisitos indispensáveis para tal objetivo. Essa situação vem mobilizando enfermeiros, gestores e pesquisadores a assumirem posturas favoráveis à implementação da $\mathrm{SAE}^{8}$.

Com o intuito em contribuir para a identificação dos diagnósticos em pacientes com risco para o surgimento de UPs, a NANDA-I incluiu um novo DE, denominado risco de UP (00249), encontrado no domínio 11, segurança/proteção, na classe 2 de lesão física. Tal DE é norteado por 42 fatores de riscos respaldados cientificamente pela National Pressure Ulcer Advisory Panel (NPUAP), que é a referência internacional para as UPs ${ }^{6,9,10}$.

Anteriormente, o DE da NANDA-I que correspondia à vulnerabilidade do paciente à UP era o risco de integridade da pele prejudicada (00047), que abordava aspectos gerais inerentes à integridade tissular. Com o novo $\mathrm{DE}$ pode-se perceber fatores específicos que colaboram para o desenvolvimento das UPs, e para a identificação destas, utilizando-se de vários instrumentos, dentre os quais se destaca a Escala de Braden (EB), que avalia o risco do paciente em desenvolver UPP ${ }^{11}$.

O objetivo do estudo foi identificar a prevalência dos fatores de risco para o DE risco de UP, de acordo com a taxonomia II da NANDA-I, em pacientes na UTI, o que possibilitará o direcionamento para uma sistematização do trabalho do enfermeiro que contribua para o cuidado prestado.

\section{MÉTODOS}

Trata-se de um estudo descritivo, transversal de natureza quantitativa, envolvendo pacientes internados na UTI, realizado em um hospital da rede privada de saúde, na cidade de Caruaru (PE), no período de janeiro a maio de 2014. No serviço pesquisado a SAE encontrava-se em fase de implementação, havia dois meses, e os DEs estavam sendo registrados pelos enfermeiros nas evoluções diárias da UTI. A população foi constituída por pacientes críticos de ambos os sexos admitidos na UTI durante a realização do estudo. Para a composição da amostra, foram adotados os seguintes critérios: ter mais de 18 anos; não apresentar UP no momento de admissão na UTI; pacientes acamados considerados críticos, permanecer na UTI por, no mínimo, 72 horas. Os critérios de exclusão foram: ser transferido para outra unidade hospitalar; obter alta da UTI; paciente evoluir a óbito.

Após aplicação dos critérios de seleção, foram recrutados 30 pacientes, que compuseram a amostra deste estudo.
A primeira parte da coleta de dados aconteceu por meio de um roteiro de entrevista segundo os domínios da taxonomia II da NANDA-I. Foram analisados os prontuários dos pacientes, os exames laboratoriais, os exames físico e clínico, além de registros no prontuário, como prescrições e evoluções médicas e de enfermagem, a fim de verificar a frequência de algum dos 42 fatores de risco publicados na NANDA-I. No entanto, para auxiliar na identificação dos fatores supracitados utilizou-se a $\mathrm{EB}$, que mostrou ser mais adequada aos pacientes do estudo. Esse instrumento determinou o risco/exposição do paciente em desenvolver UP, constados na NANDA-I.

O Termo de Consentimento Livre e Esclarecido (TCLE) foi lido e assinado por todos os pesquisados e/ou seu responsável, no qual foram assegurados o sigilo dos dados e o anonimato. $\mathrm{O}$ projeto de pesquisa foi apreciado e aprovado pelo Comitê de Ética em Pesquisa, CAAE no 22350513.6.0000.5203, em observância a Resolução 466/12, do Conselho Nacional de Saúde.

\section{RESULTADOS}

Em relação ao aspecto sociodemográfico da amostra pesquisada, dos 30 pacientes analisados, $80,0 \%$ eram do sexo feminino, e 20,0\%, do masculino. Quanto à faixa etária, $86,6 \%$ tinham idade maior que 60 anos, seguidos de 13,4\% compreendidos na faixa etária de 20 a 30 anos. Observou-se que o tempo de permanência destes na UTI variou entre 5 e 25 dias.

Após a coleta dos dados foram efetuadas algumas considerações acerca da sistematização da assistência de enfermagem com o paciente com fatores de risco para o DE risco para UP, na identificação desse DE, a ser utilizado para fomento técnico-científico no âmbito assistencial. Os 42 fatores de risco elencados para o novo DE estão descritos no Quadro 1.

A partir do julgamento clínico dos problemas reais e potenciais dos pacientes, foram estabelecidos 20 fatores de risco para o DE risco para UP, dispostos no Quadro 2, de acordo com a sua frequência, sendo comentados os de frequência superior a $50,0 \%$.

Reitera-se que os fatores relacionados ao $\mathrm{DE}$ risco para UP foram fundamentados na NANDA-I, a qual evidencia a importância da sistematização da assistência de enfermagem com a identificação de diagnósticos, definidos como 
avaliação clínica acerca das respostas do indivíduo, da família ou da comunidade aos problemas de saúde ou processos vitais, sejam eles reais ou potenciais, os quais propiciam a base para a eleição das intervenções de enfermagem para alcançar os resultados, os quais são de responsabilidade do enfermeiro $^{6}$.

O estudo possibilitou elencar 20 fatores de risco relacionados ao DE supracitado, e, em sua totalidade, apresentaram frequência superior a 50\%. Os de maior prevalência foram: escore na $\mathrm{EB}<18$, agentes farmacológicos, alteração na função cognitiva, redução na mobilidade, alteração na sensibilidade, atrito em superfície, forças de cisalhamento, pressão sobre proeminência óssea, umidade da pele, incontinência, uso de lençóis com propriedade insuficiente de redução da umidade, déficit do autocuidado, nutrição inadequada redução na perfusão tissular, os quais obtiveram prevalência de $100 \%$. Outros fatores relacionados com frequência elevada merecem destaque: doença cardiovascular $(86,6 \%)$, extremos de idade $(86,6 \%)$, gênero feminino $(80,0 \%)$, extremos de peso $(63,3 \%)$, circulação prejudicada (53,3\%) e desidratação (53,3\%).
Quadro 2. Distribuição da frequência ( $\mathrm{n}=30$ ) dos fatores de risco identificados nos participantes do estudo.

\begin{tabular}{|c|c|c|}
\hline Fatores de risco & $f$ & $\%$ \\
\hline Escore na Escala de Braden $<18$ & 30 & 100,0 \\
\hline Agentes farmacológicos & 30 & 100,0 \\
\hline Alteração na função cognitiva & 30 & 100,0 \\
\hline Redução na mobilidade & 30 & 100,0 \\
\hline Alteração na sensibilidade & 30 & 100,0 \\
\hline Atrito em superfície & 30 & 100,0 \\
\hline Forças de cisalhamento & 30 & 100,0 \\
\hline Pressão sobre proeminência óssea & 30 & 100,0 \\
\hline Úmidade da pele & 30 & 100,0 \\
\hline Incontinência & 30 & 100,0 \\
\hline $\begin{array}{l}\text { Uso de lençóis com propriedade } \\
\text { insuficiente de redução da umidade }\end{array}$ & 30 & 100,0 \\
\hline Déficit do autocuidado & 30 & 100,0 \\
\hline Nutrição inadequada & 30 & 100,0 \\
\hline Redução na perfusão tissular & 30 & 100,0 \\
\hline Doença cardiovascular & 26 & 86,6 \\
\hline Extremos de idade & 26 & 86,6 \\
\hline Gênero feminino & 24 & 80,0 \\
\hline Extremos de peso & 19 & 63,3 \\
\hline Circulação prejudicada & 16 & 53,3 \\
\hline Desidratação & 16 & 53,3 \\
\hline
\end{tabular}

Quadro 1. Fatores de risco do diagnóstico de enfermagem risco para úlcera por pressão. North American Nursing Diagnoses Association (NANDA-I, 2015).

\begin{tabular}{|c|c|c|}
\hline $\begin{array}{l}\text { 1. ADULTO: escore na Escala de } \\
\text { Braden }<18\end{array}$ & $\begin{array}{l}\text { 2. Escore }>2 \text { na classificação } \\
\text { Funcional da New York Heart } \\
\text { Association (NYHA) }\end{array}$ & 3. Linfopenia \\
\hline 4. CRIANÇA: Escala de Braden $<16$ & $\begin{array}{l}\text { 5. Escore de Classificaçăo do } \\
\text { estado físico da American Society } \\
\text { of Anesthesiologists (ASA) > } 2\end{array}$ & 6. Nutrição inadequada \\
\hline $\begin{array}{l}\text { 7. Agentes farmacológicos (p. ex., } \\
\text { anestesia geral, vasopressores, } \\
\text { antidepressivos) }\end{array}$ & $\begin{array}{l}\text { 8. Espessura reduzida da dobra } \\
\text { da pele do tríceps }\end{array}$ & 9. Pele em descamação \\
\hline 10. Alteração na função cognitiva & 11. Extremos de idade & 12. Pele ressecada \\
\hline 13. Alteração na sensibilidade & 14. Extremos de peso & $\begin{array}{l}\text { 15. Período de imobilidade } \\
\text { prolongada sobre superfície rija (p. } \\
\text { ex., procedimento cirúrgico }>2 \text { h) }\end{array}$ \\
\hline $\begin{array}{l}\text { 16. Anemia } \\
\text { 19. Atrito em superfície }\end{array}$ & 17. Forças de cisalhamento & 18. Pressão sobre proeminência óssea \\
\hline $\begin{array}{l}\text { 22. Baixo escore na escala de Risck } \\
\text { Assessment Pressure Sore (RAPS) }\end{array}$ & 23. Gênero feminino & 24. Redução na oxigenação tissular \\
\hline 25. Circulação prejudicada & 26. Hipertermia & 27. Redução na perfusãa tissular \\
\hline $\begin{array}{l}\text { 28. Conhecimento insuficiente } \\
\text { do cuidador sobre prevenção de } \\
\text { úlcera por pressão }\end{array}$ & $\begin{array}{l}\text { 29. História de acidente vascular } \\
\text { encefálico }\end{array}$ & $\begin{array}{l}\text { 30. Redução no nível de albumina } \\
\text { sérica }\end{array}$ \\
\hline 31. Déficit no autocuidado & 32. História de trauma & 33. Tabagismo \\
\hline 34. Desidratação & $\begin{array}{l}\text { 35. História de úlcera por } \\
\text { pressão }\end{array}$ & $\begin{array}{l}\text { 36. Temperatura elevada da pele em } \\
\text { torno de } 1-2^{\circ} \mathrm{C}\end{array}$ \\
\hline 37. Doença cardiovascular & 38. Imobilização física & 39. Umidade da pele \\
\hline 40. Edema & 41. Incontinência & $\begin{array}{l}\text { 42. Uso de lençóis com propriedade } \\
\text { insuficiente de redução da umidade }\end{array}$ \\
\hline
\end{tabular}




\section{DISCUSSÃO}

A EB foi construída por Braden e Bergstron, com o objetivo de diminuir a incidência de UP no serviço onde trabalha$\operatorname{vam}^{12}$. Ela foi traduzida e adaptada para a língua portuguesa por Wana Paranhos, em 1999, permitindo adequada utilização no Brasil. A EB está amparada na fisiopatologia das UPs e permite uma avaliação dos aspectos relevantes ao desenvolvimento da úlcera, segundo seis parâmetros: percepção sensorial, umidade, mobilidade e atividade, nutrição, fricção e cisalhamento. Os cinco primeiros subescores recebem uma pontuação que varia de um a quatro, enquanto fricção e cisalhamento, de um a três. A soma de cada subescore resulta na estratificação em faixas, em que os menores valores indicam as piores condições ${ }^{13}$. Assim, 100\% dos sujeitos do estudo apresentaram como fator de risco um escore na $\mathrm{EB}<18$

Neste estudo, os sujeitos, em sua totalidade, estavam expostos a agentes farmacológicos (sedativos, analgésicos, vasoativos, antibióticos, imunossupressores). Comprovadamente, esses fármacos podem ter influência direta no desenvolvimento de UP, pelas alterações sistêmicas que provocam no organismo humano. Por conseguinte, as alterações farmacodinâmicas e farmacocinéticas alteram as funções fisiológicas da pele. Diante disso, alguns remédios podem levar a imobilidade $\mathrm{e}$, consequentemente, perda da capacidade funcional, elevando, assim, o risco de o paciente desenvolver uma UP, o que requer maiores cuidados da equipe de enfermagem ${ }^{14}$.

Em decorrência de algumas patologias de origem neurológica, ocorre a diminuição do nível de consciência, mobilidade do paciente e percepção sensorial, impedindo-o de perceber a dor e desconforto ${ }^{15}$. No entanto, os pacientes que apresentam alterações cognitivas, independentemente de seu estágio, demonstram relações com o desenvolvimento de UP, uma vez que isso interfere na capacidade de se movimentar sozinho, já que não percebem a dor e/ou o desconforto ${ }^{16}$. Tais alterações estão diretamente ligadas aos fatores relacionados: função cognitiva, redução da mobilidade e alteração na sensibilidade, encontrados em 100\% dos sujeitos do estudo.

Nesse contexto, pacientes acamados com mobilidade reduzida estão sujeitos à ação de forças como atrito, pressão, cisalhamento e fricção, o que define o desenvolvimento da UP, caso não sejam adotadas medidas de prevenção que diminuam seus efeitos. Tais forças vêm sendo apontadas como um dos principais fatores extrínsecos que contribuem para o desenvolvimento das UP, uma vez que ocorrerá má perfusão tecidual, diminuindo a tolerância do tecido à pressão sobre uma proeminência óssea, fazendo com que aconteça morte celular e necrose tecidual, resultando, assim, em uma UP ${ }^{17}$. Os fatores forças de cisalhamento, pressão sobre proeminência óssea e atrito em superfície foram demonstrados em $100 \%$ dos pacientes estudados.

Ainda com relação ao nível de consciência, complicações neurológicas podem acarretar em distúrbios que resultam em uma umidade excessiva, tais como incontinências urinárias, incontinências fecais, transpiração excessiva, dentre outras. Assim, uma umidade presente por um tempo prolongado pode acarretar na maceração e ruptura da pele e, consequentemente, no desenvolvimento de uma UP ${ }^{15}$. O fator umidade da pele foi observado em $100 \%$ dos participantes deste estudo.

Outro fator encontrado no estudo foi o item incontinência (100\%). Este se relaciona diretamente com o uso de sedativos, alterações cognitivas e neurológicas. Com as alterações cognitivas e neurológicas, os pacientes não têm controle sobre seu corpo e suas necessidades fisiológicas ocorrem de forma involuntária, ficando expostos à umidade. Esta, causada pela incontinência urinária ou fecal em um paciente com déficit do autocuidado, torna-o susceptível às lesões ${ }^{15-18}$.

Segundo as recomendações da NPUAP, em relação exposição do paciente à umidade, sugere-se, além de aumentar a frequência de trocas de fraldas para pacientes com incontinência urinária e/ou fecal, utilizar dispositivos que minimizem o contato da umidade com o paciente, causada pelas incontinências. Assim, atualmente, estão disponíveis lençóis com um alto poder de absorção que podem ser descartáveis e/ou laváveis, impedindo, assim, o contato desses fluidos com a pele, o que evita a maceração, fator que contribui para o desenvolvimento das UPs ${ }^{10,16}$. Observou-se, no estudo, que $100,0 \%$ dos pacientes que apresentavam incontinências estavam utilizando lençóis com propriedade insuficiente de redução da umidade.

O déficit do autocuidado está correlacionado a alguns fatores, como: higienização corporal, manutenção da pele seca, sentar-se corretamente, realizar mudança de decúbito, higiene perineal após as eliminações e alimentar-se. Tais fatores interferem no processo de desenvolvimento das UPs; com isso, $100 \%$ da amostra apresentou déficit do autocuidado como fator de risco para o desenvolvimento de UP ${ }^{18}$.

$\mathrm{O}$ fator nutricional é um aspecto que apresenta um fator relevante no desenvolvimento dessas lesões. Todos os pacientes analisados apresentavam nutrição inadequada. Nesse contexto, 
o aporte nutricional, determinado primeiramente por uma avaliação do estado nutricional e, subsequentemente, por uma prescrição adequada para suprir as necessidades diárias de cada indivíduo de forma singular, resulta em um estado clínico ideal que colabora para a manutenção do organismo e, consequentemente, para a integridade tissular da pele, além de favorecer a regeneração e o processo cicatricial da pele ${ }^{16}$.

Assim como as drogas vasoativas, doenças cardiovasculares também interferem na perfusão tissular. A pressão capilar presente na terminação arterial da pele, em condições normais, apresenta-se em torno de $32 \mathrm{mmHg}$, e, na terminação venosa, em torno de $12 \mathrm{mmHg}$. No entanto, algumas situações podem interferir na perfusão tissular, tais como: síndrome da resposta inflamatória sistêmica (SIRS), diminuição da pressão sanguínea decorrente de alterações cardiovasculares, choque hemorrágico, choque séptico, pressão externa prolongada maior que $32 \mathrm{mmHg}$. O paciente, quando exposto a quaisquer situações dessas, sofre com a interrupção do fluxo sanguíneo para a área de atrito, a qual interfere na nutrição e oxigenação dos tecidos do local, podendo acarretar em hipóxia, isquemia, acidose tecidual, edema e necrose tissular, resultando, então, em uma UP ${ }^{19}$. Com isso, ao analisar os sujeitos do estudo foram evidenciados os seguintes fatores de risco: redução na perfusão tissular (100\%), doença cardiovascular $(86,6 \%)$ e circulação prejudicada $(53,3 \%)$.

Com o envelhecimento, aparecem as alterações e os desgastes em diversos sistemas funcionais, situações que ocorrem progressivamente e normalmente são irreversíveis. Essas alterações se tornam condições de risco para o agravamento das condições clínicas que aumentam com a idade avançada e, concomitantemente, aumentam a probabilidade do aparecimento das $\mathrm{UP}^{20}$. Neste estudo, o fator risco extremos de idade foi encontrado em $86,6 \%$ dos pesquisados.

Estudos apontam o sexo feminino como um fator de risco para o surgimento de UP, evidenciado pela presença dessas lesões predominantemente nessa população. Porém, em outros estudos realizados no Sudeste do país, verifica-se a divergência desse fator, por não encontrar explicação genética para tal ocorrência. Neste estudo, observou-se que 80,0\% dos participantes eram do sexo feminino ${ }^{21-23}$.

Em relação ao fator relacionado extremos de peso, $63,3 \%$ dos pesquisados enquadravam-se entre obesos e caquéticos. Assim, extremos de peso configura-se um fator intrínseco que deve ser levado em consideração quando se fala em desenvolvimento de UP.Pacientes obesos, geralmente, apresentam dificuldade de se mover no leito, ficando com restrição na mobilidade devido ao excesso de peso. Assim, em vez de se levantarem, estes se arrastam no leito, o que pode desencadear tais lesões. Além disso, no obeso, o fator umidade oriunda do suor também se torna um agravante, uma vez que o suor pode se alocar nas camadas de gordura da pele, provocando a maceração, o que proporciona maior risco nesses pacientes. Por outro lado, os pacientes caquéticos, por possuírem diminuição de tecido adiposo sobre as proeminências ósseas, ficam mais propensos à força de pressão sobre tais áreas ${ }^{21}$.

Alguns minerais, como o zinco, aumentam a proliferação celular e, consequentemente, a resistência do colágeno e a epitelização. Assim, o fator desidratação, encontrado em 53,3\% dos pesquisados, está relacionado com o desequilibrio hidroeletrolítico, o qual acomete a pele por meio do seu ressecamento, aumentando, assim, a suscetibilidade para o desenvolvimento das $\mathrm{UPs}^{24}$.

\section{CONCLUSÃO}

A identificação dos fatores de risco de UP reforça a importância da implantação do PE voltada a esses pacientes. Além de corroborar a formulação do DE, auxilia no planejamento das ações do enfermeiro, fundamentadas e adequadas de acordo com as necessidades de cada indivíduo.

Os resultados do estudo possibilitaram a identificação dos fatores de risco para o DE risco de UP, segundo a taxonomia II da NANDA-I.

Assim, nos 30 pacientes admitidos na UTI foram identificados 20 fatores de risco para o DE supracitado, segundo a taxonomia II da NANDA-I, cuja frequência total observada foi superior a $50 \%$.

A baixa prevalência de publicações acerca dos DEs em pacientes com risco de UP constituiu-se de uma limitação do presente estudo.

Contudo, é de suma importância o avanço de estudos relacionados à identificação dos fatores de risco para formulação dos DEs, com o intuito de direcioná-los à análise das dificuldades dos pacientes que demandam intervenções específicas de enfermagem. Dessa maneira, pode-se colaborar para o progresso científico da profissão. 


\section{REFERÊNCIAS}

1. Rogenski NMB, Kurcgant $P$. The incidence of pressure ulcers after the implementation of a prevention protocol. Rev Latino-Am Enfermagem. 2012; 20(2):333-9.

2. Silva DP, Barbosa MH, Araujo DF, Oliveira LP, Melo AF. Úlcera por pressão: avaliação de fatores de risco em pacientes internados em um hospital universitário. Rev Eletrônica Enferm. 2011;13(1):118-23.

3. Alves AGP, Borges JWP, Brito MA. Avaliação do risco para úlcera por pressão em unidades de terapia intensiva: uma revisão integrativa. J Res Fundam Care. 2014;6(2):793-804.

4. Rogenski NMB, Kurcgant P. Avaliação da concordância na aplicação da Escala de Braden inter observadores. Acta Paul Enferm. 2012;25(1):24-8

5. Brunner \& Suddarth. Tratado de Enfermagem MédicoCirúrgica. 12a ed. Rio de Janeiro: Guanabara Koogan; 2011.

6. North American Nursing Diagnoses Association (NANDA International). Diagnósticos de Enfermagem da NANDA (North American Nursing Diagnoses Association): Definições e classificação (2015-2017). Porto Alegre: Artmed; 2015.

7. Maria MA, Quadros FAA, Grassi MFO. Sistematização da assistência de enfermagem em serviços de urgência e emergência: viabilidade de implantação. Rev Bras Enferm. 2012;65(2):297-303.

8. Aiken LH, Patrician PA. Measuring organizational traits of hospitals: the Revised Nursing Work Index. Nurs Res. 2000;49(3):146-53.

9. Cruz DALM, Guedes ES, Santos MA, Sousa RMC, Turrini RNT, Maia MM. Documentação do processo de enfermagem: justificativa e métodos de estudo analítico. Rev Bras Enferm. 2016;69(1):197-204.

10. National Pressure Ulcer Advisory Panel (NPUAP). Guía Internacional. Prevenção de úlceras por pressão: Guia de referência rápida; 2014 [Internet]. [cited 2016 May 09]. Available from: http://www.npuap.org/wp-content/ uploads/2014/08/Quick-Reference-Guide-DIGITAL-NPUAPEPUAP-PPPIA-Jan2016.pdf

11. Bavaresco T, Lucena AF. Intervenções da Classificação de Enfermagem NIC validadas para pacientes em risco de úlcera por pressão. Rev Latino-Am. Enfermagem. 2012;20(6):1109-16.

12. Braden BJ, Bergstrom N. Clinical utility of Braden Scale for predicting pressure sores risk. Decubitus. 1989;2(3):44-51.

13. Freitas MC, Medeiros ABF, Guedes MVC, Almeida PC, Galiza FT, Nogueira JM. Úlcera por pressão em idosos institucionalizados: análise da prevalência e fatores de risco. Rev Gaúcha Enferm. 2011;32(1):143-50.

14. Nogueira GA, Assad LG. Avaliação de risco para úlcera por pressão: contribuição para o cuidado de enfermagem na unidade de clínica médica. Rev Enferm UFPE. 2013;7(11):6462-70.

15. Freitas JPC, Alberti, LR. Aplicação da Escala de Braden em domicílio: incidência e fatores associados a úlcera por pressão. Acta Paul Enferm. 2013;26(6):515-21.

16. Rolim JA, Vasconcelos JDMB, Caliri MHL, Santos IBDC. Prevenção e tratamento de úlceras por pressão no cotidiano de enfermeiros intensivistas. Rev Rene. 2013;14(1): 148-57.

17. Pereira AGS, Santos CT, Menegon DB, Mello BS, Azambuja F, de Fátima LA. Mapeamento de cuidados de enfermagem com a NIC para pacientes em risco de úlcera por pressão. Rev Esc Enferm USP. 2014;48(3):454-61.

18. Serpa LF, Santos VLCG, CampaniliTCGF, Queiroz M. Predictive validity of the Braden scale for pressure ulcer risk in critical care patients. Rev Latino-Am Enferm. 2011;19(1):50-7.

19. Campanili TCGF, Santos VLCG, Strazzieri PKC, Thomaz PBM, Nogueira PC. Incidência de úlceras por pressão em pacientes de Unidade de Terapia Intensiva Cardiopneumológica. Rev Esc Enferm USP. 2015;49(spe):7-14.

20. Moro A, Maurici A, Valle JB, Zaclikevis VR, Kleinubing JH. Avaliação dos pacientes portadores de lesão por pressão internados em hospital geral. Rev Assoc Med Bras. 2010;53(4):300-4.

21. Fernandes MG, Costa KN, Santos SR, Pereira MA, Oliveira DS, Brito SS. Risco para úlcera por pressão em idosos hospitalizados: aplicação da escala de Waterlow. Rev Enferm UERJ. 2012;20(1):56-60.

22. Araújo TM, Araújo MF, Cavalcante CS, Barbosa Júnior GM, Caetano JA. Acurácia de duas escalas de avaliação de risco para úlcera por pressão em pacientes críticos. Rev Enferm UERJ. 2011;19(3):381-5.

23. Fernandes NC, Torres GV. Incidência e fatores de risco de úlceras de pressão em pacientes de unidade de terapia intensiva. Rev Ciênc Cuid Saúde. 2008;7(3):304-10.

24. Andrade SDR, Santos VSARS, Souza KRF. Atuação de enfermeiros de Home Care na prevenção de úlceras por pressão. Cad Grad - Ciências Biológicas e da Saúde FACIPE. 2013;1(1):21-35. 